\title{
EDITORIAL
}

\section{Assisted ventilation as an aid to exercise training: a mechanical doping?}

\author{
N. Ambrosino
}

마 xercise training as a part of multidisciplinary pulmonary rehabilitation can improve both exercise tolerance and health-related quality of life in patients with chronic obstructive pulmonary disease (COPD) [1]. The following physiological changes contribute to these improvements: reduction of lactic acidosis; minute ventilation and heart rate for a given work rate; and enhanced activity of mitochondrial enzymes and capillary density in the trained muscles. Intensity of exercise training is of key importance. High-intensity training has improved both maximal and submaximal exercise tests and has induced both cardiorespiratory and peripheral muscle adaptations [2]. Nevertheless, in patients with severe COPD, extreme breathlessness and/or peripheral muscle fatigue may prevent higher levels of intensity training. As a consequence of flow limitation, in order to cope with the increased ventilatory demands of exercise, these patients show a breathing pattern that ultimately places greater demands on their inspiratory muscles. Indeed, in these patients, a pattern of low tidal volume and high-frequency breathing is unlikely to be advantageous, but it is the only pattern available in the presence of expiratory flow limitation and dynamic hyperinflation. Increased inspiratory muscle work may contribute to dyspnoea and exercise limitation in such patients, even before their ventilatory ceiling is attained.

To the extent that intrinsic mechanical loading and functional inspiratory muscle weakness in COPD contribute to dyspnoea, assisted ventilation should provide symptomatic benefit by unloading and assisting such overburdened ventilatory muscles. Reducing exercise dyspnoea by assisted ventilation might allow for higher levels of exercise intensity. Several studies have examined the acute effects of different modalities of ventilatory assistance on dyspnoea and exercise tolerance in advanced COPD. The message of these physiological studies could be summarised as follows: assisted ventilation delivered as noninvasive positive pressure ventilation (NPPV), either as continuous positive airway pressure (CPAP), inspiratory pressure support (IPS) or proportional assist ventilation (PAV), during exercise reduces dyspnoea and work of breathing and enhances exercise tolerance in COPD patients [3].

Therefore, there is no question of the usefulness of assisted ventilation in increasing exercise tolerance and reducing

CORRESPONDENCE: N. Ambrosino, Pulmonary Unit, Cardio-Thoracic Dept, Azienda OspedalieraUniversitaria Pisana, Via Paradisa 2, Cisanello, 57124 Pisa, Italy. Fax: 39 050996779. E-mail: n.ambrosino@ao-pisa.toscana.it dyspnoea in the acute (laboratory) setting. The following logical step is to evaluate whether assisted ventilation can be used as an aid during exercise training sessions. However, this is a different issue. Up until now, conflicting results have been reported. A randomised controlled study by BIANCHI et al. [4] found no additional benefit of assisted ventilation (in PAV modality) on exercise tolerance, dyspnoea and health status when compared with training alone. In contrast, HAWKINS et al. [5] evaluated the effects of providing PAV to patients with more severe COPD during a supervised high-intensity outpatient cycle exercise programme. After ventilation-assisted training, mean training intensity and peak work rate were higher in the group that used PAV. Iso-workload lactataemia after training was reduced in the assisted group more than in the unassisted group. A significant inverse relationship was found between reduction in iso-workload lactataemia after training during the constant work rate test and peak work rate achieved during the last week of training.

In a randomised controlled study published in this issue of the European Respiratory Journal, VAN 'T HUL et al. [6] have evaluated the effects of training with mask IPS in COPD patients. These authors compared $10 \mathrm{cmH}_{2} \mathrm{O}$ IPS (IPS10) with $5 \mathrm{cmH}_{2} \mathrm{O}$ IPS (IPS5; a level of assistance they considered as "sham") delivered during high-intensity training. IPS10 resulted in significantly larger improvements in exercise performance than training with IPS5; therefore, the authors concluded that IPS10 may be considered as adjunct during high-intensity exercise training.

VAN 'T HUL et al. [6] have done their best to demonstrate the effectiveness of NPPV as an aid to exercise training in COPD patients. Nevertheless, this study raises some concerns and does not solve the problems regarding the practical use of NPPV in a routine setting. A limitation of this study is the lack of blindness of the researchers to the treatment allocation, which is also true for other "positive" studies [5]. Indeed, other studies have shown differences in intensity of training (as compared with baseline peak power) obtained with training in spontaneous breathing. For instance, the intensities obtained in this and other studies [4-7] were greater than those reported in a study by MALTAIS et al. [8]. These differences may be explained by the varying levels of encouragement given by researchers during training and/or assessment of exercise tolerance. In this respect, the lack of blindness of this study is a major drawback.

Another practical issue must be considered: the ventilator. Commercial "home" ventilators may be used with some 
success in the treatment of acute respiratory failure due to COPD exacerbations [9], but their use when patients require high ventilatory levels, such as in most severe conditions or during exercise, is rather questionable. Conversely, an "intensive care unit" ventilator, such as that used in the study by VAN 'T HUL et al. [6], may not be the most convenient and cheapest device in a routine setting. Would we need such expensive ventilators for each of the patients who were presumably submitted to group training sessions?

In contrast to previous studies [4, 5], VAN 'T HUL et al. [6] have used IPS as a modality of NPPV. IPS is a form of mechanical ventilation that can effectively assist ventilation when applied noninvasively to patients in acute and chronic respiratory failure. It is a pressure-targeted mode in which each breath is patient-triggered and supported [10]. It provides breath-bybreath ventilatory support by means of a positive-pressure wave that is synchronised with the inspiratory effort of the patient. During inspiration, the airway pressure is raised to a pre-set level: the pressure support level. This level is maintained until the machine determines the end of a patient's respiratory effort or detects a patient's demand for expiration. Previous studies have delivered PAV during training [4, 5]. PAV is a mode of partial ventilatory assistance endowed with characteristics of proportionality and adaptability to the intensity and timing of spontaneous ventilatory pattern by providing inspiratory flow and pressure in proportion to the patient's effort. The ventilator delivers pressure according to the motion equation, generating a pressure that is in proportion to a patient's spontaneous effort. A portion of the total mechanical workload, i.e. elastance and resistance, is taken over according to a level of assistance, which has been decided by the caregiver and can specifically unload the resistive burden (flow assist) and the elastic burden (volume assist) [11].

No information on whether an external positive end-expiratory pressure (PEEP) was added by VAN 'T Hul et al. [6]. It should be considered that patients' respiratory mechanics can change during exercise, for example due to the increase of intrinsic PEEP (PEEPi) [12], thus profoundly affecting the effect of NPPV. A change in PEEPi may affect the observed influence of NPPV because any inspiratory pressure assist is not initiated until PEEPi is overcome, which is why exercise improves when NPPV is superimposed upon CPAP/PEEP. CPAP/PEEP compensates for the threshold load, whereas NPPV compensates the elastic and resistive load of inspiration.

The use of NPPV during training may have a limited role on a routine basis. An uncertainty regarding the practical applicability of the results from the study by VAN'T HUL et al. [6] and another study [5] is the use of face mask or mouthpiece. During high-intensity exercise, patients may breathe through the mouth rather than the nose, thus requiring a face mask or mouthpiece. Nevertheless, compliance to a face mask may be not as easy; however, in the treatment of acute exacerbations of COPD, both nasal and face masks were equivalent in term of effectiveness [13]. It should be considered that the expectations and mood of a patient in the intensive care unit, fighting for their life, or those of a randomised study patient in the laboratory, supported by the enthusiasm of the operator, may not be the same as a relatively stable, although dyspnoeic, patient participating a routine training session.
Many COPD patients have significant comorbidity, including a high proportion of cardiac ischaemic disease. It is of concern that reduction of dyspnoea with this kind of mechanical doping might expose an unaware COPD ischaemic patient to a load greater than his/her ischaemic threshold. In the study by BIANCHI et al. [4], a high rate of dropouts due to lack of compliance in the ventilated group, and a mean time of $17 \mathrm{~min}$ spent to set the ventilator and to supervise the training session during NPPV were practical drawbacks of the addition of mask ventilation during a high-intensity training programme. These results indicated that it is not worthwhile to submit patients to such equipment where mask and other related troubles are experienced, given the need for constant supervision by an individual operator to check for leaks, to reset the ventilator when needed and a substantial risk of lack of compliance. Furthermore, this indicates a need for a one-to-one patient/therapist ratio, which will add to the cost of rehabilitation programme.

Any conclusion about the potential clinical utility of ventilatory assistance during training is difficult because of the small study sample size, variability of pathophysiological abnormalities at study entry, differences in the ventilatory devices and operating characteristics of the ventilators, differences in pressure-optimisation protocols or lack of optimisation, differences in breathing-circuit design with the potential for carbon dioxide rebreathing, and differences in exercise protocols and evaluative methods of dyspnoea. Larger prospective controlled studies should be required to determine if ventilatory assistance will eventually be a useful adjunct to standard exercise protocols for dyspnoeic patients with more advanced cardiopulmonary diseases. As in any study, large or small, a thorough description of subjects is required; the ability of the ventilatory device to meet the demands of the patient must be demonstrated; the operating characteristics must be described; and carbon dioxide rebreathing must be within acceptable limits. The study by VAN 'T HUL et al. [6] may stimulate further research with additional optimism.

\section{REFERENCES}

1 Troosters T, Casaburi R, Gosselink R, Decramer M. Pulmonary rehabilitation in chronic obstructive pulmonary sisease. Am J Respir Crit Care Med 2005; 172: 19-38.

2 Casaburi R, Patessio A, Ioli F, Zanaboni S, Donner CF, Wasserman K. Reduction in exercise lactic acidosis and ventilation as a result of exercise training in patients with obstructive lung disease. Am Rev Respir Dis 1991; 143: 9-18.

3 Ambrosino N, Strambi S. New strategies to improve exercise tolerance in COPD. Eur Respir J 2004; 24: 313-322.

4 Bianchi L, Foglio K, Porta R, Baiardi R, Vitacca M, Ambrosino N. Lack of additional effect of adjunct of assisted ventilation to pulmonary rehabilitation in mild COPD patients. Respir Med 2002; 96: 359-367.

5 Hawkins P, Johnson LC, Nikoletou D, et al. Proportional assist ventilation as an aid to exercise training in severe chronic obstructive pulmonary disease. Thorax 2002; 57: 853-859.

6 Van 't Hul A, Gosselink R, Hollander P, Postmus P, Kwakkel G. Training with inspiratory pressure support in 
patients with severe chronic obstructive pulmonary disease. Eur Respir J 2006; 27: 65-72.

7 Emtner M, Porszasz J, Burns M, Somfay A, Casaburi R. Benefits of supplemental oxygen in exercise training in non-hypoxemic COPD patients. Am J Respir Crit Care Med 2003; 68: 1034-1042.

8 Maltais F, LeBlanc P, Jobin J, et al. Intensity of training and physiologic adaptation in patients with chronic obstructive pulmonary disease. Am J Respir Crit Care Med 1997; 155: 555-561.

9 Ambrosino N, Corrado A. Obstructive pulmonary disease with acute respiratory failure. In: Muir JF, Simonds AK, Ambrosino N, eds. Noninvasive mechanical ventilation. Eur Respir Mon 2001; 16: 11-32.
10 Brochard L. Pressure support ventilation. In: Tobin MJ, ed. Principles and practice of mechanical ventilation. New York, McGraw-Hill Inc., 1994; pp. 239-256.

11 Bianchi L, Foglio K, Pagani M, Vitacca M, Rossi A, Ambrosino N. Effects of proportional assist ventilation on exercise tolerance in COPD patients with chronic hypercapnia. Eur Respir J 1998; 11: 422-427.

12 Petrof BJ, Calderini E, Gottfried SB. Effect of CPAP on respiratory effort and dyspnoea during exercise in severe COPD. J Appl Physiol 1990; 69: 179-188.

13 Kwok H, McCormack J, Cece R, Houtchen J, Hill NS. Controlled trial of oronasal versus nasal mask ventilation in the treatment of acute respiratory failure. Crit Care Med 2003; 31: 468-473. 